\title{
Retos de la Universidad Michoacana de San Nicolás de Hidalgo ante los estudiantes con discapacidad
}

\section{Challenges of the Universidad Michoacana de San Nicolás de Hidalgo to students with disabilities}

\author{
KAREN LizetTe Cíntora OSEguera* \\ MARÍA DE LOURDES VARGAS GARDUÑO** \\ FABIOLA GoNZÁlEZ BETANZOS***
}

El objetivo del proyecto que dio origen al artículo fue visibilizar y recuperar las voces de los estudiantes en situación de discapacidad, así como formar tutores especializados en la atención a este sector poblacional en una universidad de México. El proyecto inició con la identificación de aspirantes con discapacidad en la encuesta de ingreso y continuó en una segunda etapa de formación de tutores en cada facultad. Trabajamos con 59 docentes tutores en talleres sobre educación inclusiva, en los que se analizaron las barreras a las que se enfrentan los estudiantes en situación de discapacidad. Las historias de vida de estudiantes con discapacidad nos permitieron reconocer algunos elementos facilitadores que favorecen su trayectoria en el contexto universitario. Las conclusiones apuntan a que aún debe recorrerse un largo camino para llevar a cabo los procesos de gestión que faciliten generalizar la atención a la discapacidad en las instituciones de educación superior y, en particular, en la Universidad Michoacana de San Nicolás de Hidalgo.

The objective of the project that gave rise to the article was to make visible and recover the voices of students with disabilities, as well as to train tutors specialized in supporting this sector of the population. The project began with the identification of applicants with disabilities from the entrance survey of the 2017-2018 school year and a second stage of tutor training in each university department. We worked with 59 teacher tutors in workshops on inclusive education, in which an analysis was made about the barriers faced by students with disabilities. Likewise, the life stories of students with disabilities allowed us to identify some facilitating elements that assist their trajectory in the university context. It is concluded that there is still a long way to go to carry out the management processes that allow generalizing the attention to disabilities in higher education institutions and, in particular, within the Universidad Michoacana de San Nicolás de Hidalgo.

\author{
Palabras clave: \\ educación \\ superior, \\ discapacidad, \\ educación \\ inclusiva, \\ tutoría, \\ barreras y \\ facilitadores
}

\section{Keywords:}

higher
education,
disability,
inclusive
education,
tutoring,
barriers and
facilitators

Recibido: 16 de febrero de 2019. | Aceptado para su publicación: 31 de julio de 2019. Recuperado de: https://sinectica.iteso.mx/index.php/SINECTICA/article/view/960 doi: 10.31391/S2007-7033(2019)0053-006

Sección: Investigaciones temáticas

\footnotetext{
* Licenciada en Psicología y licenciada en Educación Especial con Especialidad en Discapacidad Intelectual. Docente del Centro Psicopedagógico de Educación Especial de La Piedad, Michoacán. Cursa la maestría en Psicología en la Universidad Michoacana de San Nicolás de Hidalgo (UMSNH). Correo electrónico: kcintorapsic@gmail.com

${ }^{* *}$ Doctora en Ciencias Sociales, con especialidad en Psicología Social. Pertenece al SNI, con nivel 1. Profesora-investigadora de la Facultad de Psicología de la UMSNH y coordinadora del cuerpo académico consolidado "Educación, cultura y procesos de aprendizaje". Líneas de investigación: educación intercultural bilingüe, alfabetización académica, formación docente. Correo electrónico: luluvargas61@gmail.com/ http://orcid.org/0000-0002-7384-3098

*** Doctora en Metodología en Ciencias del Comportamiento y de la Salud. Pertenece al SNI, con nivel 1. Profesora-investigadora de la Facultad de Psicología de la UMSNH y pertenece al cuerpo académico consolidado "Intervenciones psicológicas y socioculturales en familia, género, migración, educación y salud". Líneas de investigación: creación de instrumentos de medición, análisis de datos, desarrollo de modelos psicométricos y estadísticos para el estudio de las trayectorias escolares y laborales, así como investigación en ciencias de la salud. Correo electrónico: fbetanzos@umich.mx/http:// orcid.org/0000-0003-4585-7211
} 


\section{INTRODUCCIón}

Las universidades también tienen la posibilidad de participar en la formación cultural e ideológica de la sociedad $y$, con ello, en la formación de valores, actitudes y representación de la propia sociedad.

Es esta una responsabilidad de las universidades, tengan o no conciencia y voluntad de ello.

ANUIES, 2002

E n México, existen 7.8 millones de habitantes que presentan algún tipo de discapacidad, según datos de la Encuesta Nacional de la Dinámica Demográfica 2018. Por entidad federativa, las mayores prevalencias de discapacidad se ubican en Zacatecas (9.6\%), Tabasco (9.4\%), Guerrero (8.7\%), Michoacán de Ocampo (8.6\%), Veracruz (8.2\%) y Colima (8.1\%) (INEGI, 2019). En cuanto a la participación en el ámbito educativo, la misma encuesta, pero de 2014 (INEGI, 2016), señala que el $60.6 \%$ de las personas de 3 a 29 años de edad que no presentan discapacidad ni limitación asisten a la escuela. De la población en situación de discapacidad de la misma edad, solo el $46.5 \%$ se forma en una escuela, por lo que existe una brecha de 14.1 puntos porcentuales entre los dos tipos de población.

Dicha encuesta también reporta que las personas en situación de discapacidad con los porcentajes más altos de asistencia escolar son las que se encuentran en edad de asistir a la primaria (6-11 años) o a la secundaria (12-14 años); la asistencia escolar disminuye notablemente a partir de los 19 años, es decir, en la educación superior. Otro dato importante apunta que el porcentaje de la población analfabeta de 15 años y más en situación de discapacidad es del 22.7, que supera por mucho a la de la población sin discapacidad ni limitación que también es analfabeta (3.8\%). En las cifras anteriores apreciamos el enorme rezago educativo de las personas en situación de discapacidad.

En México existe todo un marco normativo que garantiza el respeto al derecho a la educación de las ciudadanas y los ciudadanos mexicanos, que, en lo que concierne a las personas en situación de discapacidad, está sustentado en las siguientes leyes: artículo 3ำ de la Constitución Mexicana; artículo 24 de la Convención sobre los Derechos de las Personas en Situación de Discapacidad; artículo 41 de la Ley General de Educación; artículo 1ํ de la Ley General de las Personas en Situación de Discapacidad, y en la Ley Federal para Prevenir y Eliminar la Discriminación, entre otros. Empero, lo cierto es que el sistema educativo nacional no ha sido capaz de garantizar la cobertura y ofrecer servicios educativos de calidad con sentido de equidad en todos los niveles educativos (Juárez, 2015):

De este modo, el derecho a la educación de las personas en situación de discapacidad se ha centrado en los niveles educativos básicos "y en tiempo reciente, con la obligatoriedad de la educación media superior, también se ha extendido a este nivel" (Méndez, 2015, p. 4). No obstante, "la educación superior ha estado en muchas ocasiones vetada” (Novo y Muñoz, 2012, p. 105) para este sector poblacional, como lo mencionan Cruz y Casillas (2017).

Sin embargo, con la masificación de la educación superior y los cambios en la cultura y atención a la discapacidad, generadas por las transformaciones en las políticas educativas 
internacionales y nacionales, se ha dado un incremento significativo en el número de estudiantes en situación de discapacidad, que aspiran a cursar estudios universitarios (Méndez, 2015).

A la par, la educación superior pasa un momento de cambio "marcado por la coyuntura que lleva a replantear el rol de la universidad, que ya no solo se centra exclusivamente en la docencia y en la investigación, sino también en la generación de valor social" (Fundación Universia, 2016, p. 5). De ahí que, en la actualidad, las instituciones de educación superior (IES) deban cumplir con una serie de estándares de calidad, y uno de ellos es la atención a los estudiantes en situación de discapacidad. Por lo tanto, se está ante una transición importante de tal modo que la universidad se constituya en un espacio para la igualdad, donde se considere la individualidad de manera no discriminatoria y se valore en forma positiva la diversidad humana (Álvarez, 2012), dada la trascendencia del rol de las universidades como organizaciones inclusivas para promover la superación de situaciones de exclusión (Gairín y Suárez, 2016).

La Universidad Michoacana de San Nicolás de Hidalgo (UMSNH) es la IES más antigua de México y la primera universidad autónoma de América. Sus antecedentes históricos se remontan a 1540, año en que don Vasco de Quiroga fundó el Colegio de San Nicolás Obispo; sin embargo, es en 1917 cuando el ingeniero Pascual Ortiz Rubio la constituye como universidad. En la actualidad, es la máxima casa de estudios del estado de Michoacán (Mercado-Vargas, Mercado-Flores y Mercado-Flores, 2016).

Desde su fundación, hace más de cien años, la UMSNH se ha caracterizado por ser una noble institución con una filosofía humanista y un gran sentido de responsabilidad social. En su Plan de Desarrollo Institucional 2010-2020 se plantea ofrecer una educación de calidad que, de acuerdo con los planteamientos de la Organización de las Naciones Unidas para la Educación, la Ciencia y la Cultura (Unesco), deberá fundarse en tres ejes rectores: una mayor pertinencia, una mayor equidad en el acceso y los resultados, y el respeto a los derechos de la persona.

De igual modo, asume la encomienda de "hacer de la educación superior un bien público, a través de esfuerzos que permitan una educación incluyente, que garantice la equidad y promueva la movilidad social, favoreciendo la formación integral de los estudiantes, y su participación activa en esquemas de desarrollo en aras del bienestar colectivo" (UMSNH, 2010, p. 16). No obstante, en las metas planteadas aún no existe ningún documento oficial que permita la operacionalización de tal encargo a fin de atender la diversidad, específicamente, al estudiantado en situación de discapacidad. Vargas-Garduño, Castro y Méndez (2017) señalan que la UMSNH tiene mucho que trabajar al respecto, y comenzar por la identificación de la población con requerimientos especiales.

El problema en el nivel de educación superior inicia con la falta de información estadística o cualitativa sobre los estudiantes con alguna discapacidad. Aunque el contar con estos datos no garantiza la implementación de acciones pertinentes al respecto, es un hecho que la ausencia de información hace más difícil que se tomen en cuenta sus necesidades específicas y, por ende, la promoción de condiciones de equidad que favorezcan su desarrollo educativo. En consecuencia, el estudiantado en situación de discapacidad se encuentra inmerso en procesos de invisibilidad, exclusión y no reconocimiento. De este modo, se pone en evidencia la gran brecha que existe entre el discurso normativo y las prácticas reales en la UMSNH. 
La Unesco (2005) plantea que la educación inclusiva debe ser entendida como un proceso que permite abordar y responder a la diversidad de las necesidades de los estudiantes a partir de una mayor participación en los aprendizajes, las actividades culturales y comunitarias, así como la reducción de la exclusión dentro y fuera del sistema educativo. De ahí que, para lograr una educación inclusiva, no basta solo con permitir el acceso de los estudiantes en situación de discapacidad a la universidad, como lo mencionan Castellana y Salas (2005), sino que se requieren adaptaciones y atenciones personalizadas por parte de la comunidad universitaria.

\section{ACCIONES RELEVANTES TENDENTES A LA EDUCACIÓN INCLUSIVA EN INSTITUCIONES DE EDUCACIÓN SUPERIOR}

Algunas IES que están implementando políticas y desarrollando programas para favorecer la educación inclusiva de estudiantes en condición de discapacidad son: Universidad de Guadalajara, Universidad Autónoma del Estado de Morelos, Universidad Autónoma de Sinaloa, Universidad Autónoma del Estado de Hidalgo, Universidad Nacional Autónoma de México, Universidad Autónoma de Nuevo León, Universidad Autónoma de Coahuila, Universidad de Colima, Universidad Autónoma de Guerrero, Universidad Autónoma de Tlaxcala, Universidad Autónoma de San Luis Potosí, Universidad Veracruzana y Universidad Autónoma de Tamaulipas.

Las acciones que están llevando a cabo algunas de estas universidades se han clasificado como "acciones para el ingreso", "acciones para la permanencia" y "acciones para el egreso", por su relación con las fases en la trayectoria de los estudiantes en la institución, y se añade el apartado "otras acciones", las cuales, sumadas a las anteriores, contribuyen al desarrollo de la educación inclusiva para estudiantes en situación de discapacidad en IES.

En relación con el proceso de ingreso, encontramos que las universidades prestan atención a dos aspectos específicos: accesibilidad web y adaptaciones para la realización del examen de ingreso (Camacho y Navarro, 2011; Romero, Kitaoka y Rodríguez, 2014). Una vez que los alumnos con discapacidad ingresan, las IES promueven la permanencia mediante tutorías, accesibilidad arquitectónica y uso de tiflotecnología, entendida como el conjunto de técnicas, conocimientos y recursos encaminados a procurar a las personas con ceguera o deficiencia visual los medios oportunos para la correcta utilización de la tecnología (ONCE, 2019; Guajardo-Ramos, 2017; Romero, Kitaoka y Rodríguez, 2014).

En el egreso, las IES se enfocan en la inclusión laboral de los estudiantes con discapacidad (Programa Erasmus, 2016). Otras acciones para el logro de una cultura y prácticas inclusivas son el levantamiento de un censo (Méndez, 2015); la creación en las universidades de licenciaturas o maestrías encaminadas a la inclusión de las personas en situación de discapacidad y proyectos de investigación generados por cuerpos académicos (Cruz y Casillas, 2017; Guajardo-Ramos, 2017; Méndez, 2015), así como la organización de foros y coloquios sobre el tema. 


\section{ESTRATEGIA METODOLÓGICA}

Nuestra investigación partió de la lógica de la investigación-acción, cuya intención transformadora resultó pertinente gracias a las tres fases desarrolladas: identificación de aspirantes en situación de discapacidad, formación de tutores e intervención con estudiantes con discapacidad a través de "historias de vida".

Para la identificación de aspirantes en situación de discapacidad que ingresarían a la UMSNH durante el semestre 2017-2018, contamos con el apoyo de la Coordinación de Responsabilidad Social y Formación Docente de la UMSNH, a través de la cual solicitamos al área responsable de la ficha de preingreso la inclusión de preguntas que permitieran recabar datos relevantes sobre esos jóvenes; no obstante, la información obtenida resultó imprecisa, por lo que fue necesario establecer un protocolo de llamadas telefónicas para determinar las características de la discapacidad. Posteriormente, sistematizamos los datos mediante la estadística descriptiva para conocer, de manera general, sus características.

Diseñamos e implementamos el taller "Introducción a la educación inclusiva", impartido a docentes tutores interesados en formarse para ofrecer una atención equitativa a personas con discapacidad, con el apoyo de una profesora externa a la UMSNH. Contamos con la participación de 59 docentes tutores, distribuidos en dos grupos: el primero conformado por 35 tutores (octubre de 2018) y el segundo por 24 (noviembre de 2018). Realizamos observación participante durante la impartición de ambos talleres y analizamos los productos generados durante estos, principalmente, los ensayos finales.

En la intervención con estudiantes con discapacidad a través de la historia de vida participaron cinco estudiantes de licenciatura (Derecho, Psicología, Enfermería, Arquitectura e Informática Administrativa) de primero a quinto año, de los cuales dos eran mujeres (discapacidad visual, discapacidad auditiva) y tres, hombres (discapacidad motriz y discapacidad visual).

\section{ALgunOS DATOS RELEVANTES DE LOS ESTUDiAnTES DE NUEVO INGRESO}

Los estudiantes en situación de discapacidad identificados son 20, de los cuales el $40.0 \%$ son mujeres $(n=8)$ y el $60.0 \%$, hombres $(n=12)$. En cuanto al nivel escolar, dos estudiantes cursan sus estudios de preparatoria, 17, de licenciatura y uno de posgrado. En la tabla 1 se presentan las frecuencias y los porcentajes de alumnos por programa educativo; los datos indican que existe una mayor incidencia en el nivel de licenciatura (85.0\%), y las carreras con mayor índice de prevalencia son Bellas Artes (29.41\%) y Derecho (29.41\%). La sumatoria de ambas representa el $58.82 \%$, y se distinguen del resto de la elección en otras carreras. 
Tabla 1. Distribución de la muestra por carrera del nivel de licenciatura

\begin{tabular}{|c|c|c|}
\hline Programa educativo & Total & $\%$ \\
\hline Bellas Artes & 5 & 29.41 \\
\hline Derecho & 5 & 29.41 \\
\hline Enfermería & 1 & 5.88 \\
\hline Odontología & 1 & 5.88 \\
\hline Psicología & 1 & 5.88 \\
\hline Arquitectura & 1 & 5.88 \\
\hline Informática Administrativa & 1 & 5.88 \\
\hline Ingeniería en Computación & 1 & 5.88 \\
\hline Ingeniería en Mecatrónica & 1 & 5.88 \\
\hline Total & 17 & 100 \\
\hline
\end{tabular}

En cuanto a la variable clínica (ver tabla 2), los datos revelan que el tipo de discapacidad más frecuente es la motriz (parálisis cerebral, espina bífida, malformación congénita y amputación de extremidades), con un $60.0 \%$, seguido de la discapacidad visual (ceguera y baja visión), discapacidad auditiva (pérdida auditiva), discapacidad intelectual y otras (trastorno del espectro autista), con $10.0 \%$.

Tabla 2. Distribución de la muestra por tipo de discapacidad

\begin{tabular}{|c|c|c|}
\hline Tipo de discapacidad & Total & $\%$ \\
\hline Discapacidad motriz & 12 & 60.0 \\
\hline Discapacidad visual & 2 & 10.0 \\
\hline Discapacidad auditiva & 2 & 10.0 \\
\hline Discapacidad intelectual & 2 & 10.0 \\
\hline Otras & 2 & 10.0 \\
\hline Total & 20 & 100 \\
\hline
\end{tabular}

A partir de estos resultados, es posible identificar el nivel de licenciatura como el de mayor número de estudiantes en situación de discapacidad matriculados en la UMSNH, y las carreras de Bellas Artes y Derecho como las de mayor predilección por parte de este grupo poblacional. También es de mencionar la discapacidad motriz como la de mayor prevalencia. Además, existe una diferencia entre el número de mujeres en situación de discapacidad matriculadas en la universidad en comparación con el de hombres. Es visible, asimismo, el escaso número de estudiantes en situación de discapacidad que cursan el bachillerato y el posgrado en la UMSNH.

\section{IDENTIFICACIÓN DE BARRERAS A PARTIR DE LOS TALLERES “INTRODUCCIÓN A LA EDUCACIÓN INCLUSIVA"}

El análisis de los datos generados durante los talleres y, en especial, en los ensayos que constituyeron el producto final se centró en la identificación de barreras que 
enfrentan los estudiantes en situación de discapacidad dentro de la UMSNH. Las barreras son todos aquellos factores en el entorno de una persona que, cuando están presentes o ausentes, limitan el funcionamiento y producen discapacidad (OMS, 2001) o dificultan la funcionalidad de personas en condición de discapacidad. Con base en este concepto, construimos seis categorías: barreras físicas y arquitectónicas; barreras en los procesos de selección; barreras para la comunicación; barreras metodológicas y didácticas; y barreras actitudinales y en la tutoría.

\section{Barreras físicas y arquitectónicas}

Diversas son las dificultades que deben sortear los estudiantes en situación de discapacidad que deciden ingresar a la universidad; las barreras físicas y arquitectónicas son las que podemos encontrar con más facilidad. Los tutores participantes en los talleres evidenciaron su existencia en las instalaciones y los edificios de la UMSNH:

... dentro de la Facultad de Químico-Farmacobiología hay siete edificios [...] en ninguno de estos edificios se cuenta con rampas que faciliten el acceso a estudiantes que presentan dificultades (AL-ensayo).

El Colegio Primitivo y Nacional de San Nicolás de Hidalgo [...] es una institución que cuenta con enormes escaleras mismas que no tienen acceso para estudiantes discapacitados (SG-ensayo).

En el Colegio de San Nicolás [...] en dado caso que existiera algún estudiante con discapacidad auditiva les comentaré que el edificio es muy alto y los trechos de las aulas también y, por lo tanto, la acústica no es nada favorable porque se hace mucho eco (RG).

Únicamente tenemos rampas pero no contamos con baños acondicionados para la discapacidad motriz (CM-ensayo).

La UMSNH, por su antigüedad, alberga edificios históricos, algunos incluso considerados patrimonio de la humanidad, los cuales deben respetar ciertos lineamientos para que no se demerite el valor histórico y cultural del edificio. No obstante, ningún edifico histórico debe estar por encima de un derecho humano fundamental, como la educación. Por ello, uno de los principales retos que debe enfrentar la UMSNH es lograr que sus instalaciones sean físicamente accesibles para todos. Entendemos la accesibilidad como la posibilidad de acceso a todos los potenciales usuarios sin excluir a aquellos con limitaciones individuales (González y Farnós, 2009).

\section{Barreras en los procesos de selección}

En la educación media y superior, los procesos de selección se constituyen en una limitante para el acceso de los estudiantes en situación de discapacidad; lo anterior queda manifestado en los siguientes comentarios:

En los procesos de selección generalmente quedan en desventaja estudiantes que han enfrentado barreras en el aprendizaje en etapas anteriores de su formación (DT- ensayo).

Los programas para nuevo ingreso de las IES están plagados de contradicciones y obstáculos burocráticos [...] desde el ingreso, se halla sometido o excluido el estudiante (OR-ensayo). 
Los procesos de selección para ingresar a los posgrados son rigurosos y están determinados por la normativa de la propia universidad y en gran medida por los estándares impuestos (DV).

Alcantud (1995) considera que el acceso a la enseñanza superior por parte del estudiantado en situación de discapacidad se ve afectado por una gran cantidad de procesos selectivos a los que se ven sometidos, sin que en verdad se tengan en cuenta, en muchos casos, las características y necesidades específicas de estos estudiantes. De ahí que otro de los retos para la UMSNH es la adecuación de sus procesos de admisión, y la modificación de su carácter exclusivamente selectivo para convertirlos en un elemento diagnóstico que permita identificar las áreas de oportunidad en los alumnos e implementar acciones que los ayuden a desarrollarlas.

\section{Barreras para la comunicación}

Educar en la diversidad supone crear condiciones de equidad; para lograrlo, se hace necesario minimizar todas aquellas barreras comunicacionales que limitan la plena inclusión de los estudiantes en situación de discapacidad. Al respecto, los participantes compartieron sus apreciaciones:

No contamos con libros en sistema Braille, ningún profesor sabemos el idioma de señas (CM-ensayo).

La UMSNH actualmente tiene sesgos y retraso con la inclusión de la lengua de señas mexicana (LSM) en la educación media superior no hay intérpretes, lo que hace que la continuidad de los estudios se vea truncada para aquellas personas que no tienen recursos económicos para continuar (AZ-ensayo).

La UMSNH aún carece de sensibilización sobre el tema [...] en la UMSNH no se han generado cambios en sus políticas institucionales. Obligando a la comunidad sorda a mantenerse marginada por la falta de acceso (AZ-ensayo).

Muchas IES, al igual que la UMSNH, no cuentan con recursos educativos (libros en braille, audiolibros, etcétera) adaptados o con los apoyos específicos (intérpretes de lengua de señas mexicana) para dar respuesta a las demandas particulares del estudiantado en situación de discapacidad. Sin embargo, se deben gestionar los recursos necesarios para resolver estas limitaciones y minimizar este tipo de barreras.

\section{Barreras metodológicas y didácticas}

La educación en y para la diversidad está exigiendo un nuevo profesor universitario, un docente capacitado cuya actitud ante la diversidad, su formación, los recursos y apoyos que reciba adquieren especial relevancia (Álvarez, 2017). En este sentido, los tutores mencionaron:

Los maestros no sabían de su condición por lo tanto el proceso de enseñanza-aprendizaje no estaba siendo el óptimo (ZF-ensayo).

En mi clase tuve a una chica hipoacúsica, pero me di cuenta hasta un mes después de que habíamos iniciado el curso, cuando ella se me acercó y me dijo que por favor no 
caminara por los pasillos del salón, porque ella tenía dificultades auditivas, pero había aprendido a leer los labios; pero si yo me la pasaba caminando, ella no podía ver mis labios y no escuchaba lo que yo decía... (VG, oral).

La educación inclusiva requiere procesos formativos flexibles: mientras más variados sean los materiales curriculares, se emplearán más canales para transmitir la información, las actividades de aprendizaje serán diversas, el estudiantado contará con más recursos que le faciliten el acceso y, por ende, se motivarán más para aprehender los conocimientos que se proponen. Esto resulta significativo debido a que, en la actualidad, atender la diversidad del estudiantado universitario es una competencia que los docentes han de desarrollar como indicador de su excelencia profesional y calidad docente (Álvarez, 2017).

\section{Barreras actitudinales}

Álvarez (2017) menciona que las barreras más difíciles de sortear son invisibles y están en las personas que integran la comunidad universitaria. Los tutores identificaron la actitudinales:

Los estudiantes con discapacidad sufren en algunos casos de violencia física y emocional por parte del resto de la población, lo cual les obstaculiza su formación académica (docente AH).

En la institución existe segregación de estudiantes, ya sea por su nivel socioeconómico, raza, sexo, religión, capacidades físicas o intelectuales [...] lo cual da como resultado un bajo rendimiento académico y fomenta un ambiente propenso a la discriminación y de ataque a los estudiantes vulnerables (docentes RO y SS, ensayo).

A lo largo de los siete años de ejercicio docente en la Facultad de Arquitectura de la UMSNH, se presentan grupos de alumnos cada vez más segregados, poco integrados y cooperativos, integrantes egoístas e individualistas (HG-ensayo).

Los discursos anteriores permiten apreciar que los estudiantes en situación de discapacidad no son bien acogidos dentro de la UMSNH, pues suelen ser segregados, discriminados e incluso sufrir violencia. En este ámbito, la UMSNH, al igual que muchas otras IES, tiene mucho que trabajar para crear una verdadera cultura inclusiva, que valore positivamente la diversidad. El principio de que la diferencia enriquece es central en la idea de la educación inclusiva (Moriña, 2004). Según Méndez (2015) y Del Olmo (2005), no es en la homogeneidad donde se producen los aprendizajes más significativos, sino en la heterogeneidad.

Para ir construyendo una verdadera educación inclusiva, es indispensable comenzar por el cambio de actitud hacia la diversidad. La presencia de estudiantes con características diversas favorece el desarrollo de aprendizajes no solo en el ámbito académico, sino, sobre todo, en el fomento de valores como respeto, tolerancia, colaboración y democracia: la inclusión es "una de las bases para las sociedades democráticas y uno de los valores que, junto a la interculturalidad, contribuyen a la creación de una nueva escuela y una mejor sociedad" (Moliner, 2008, p. 30). Por ende, la educación inclusiva y, por lo tanto, las escuelas y universidades inclusivas benefician a las personas con discapacidad y también a todos los estudiantes en general (Moriña, 2008; Esteban-Guitart, Rivas y Pérez, 2012), ya que la enseñanza es más eficaz cuanto más inclusiva es la institución. 


\section{Barreras en la tutoría}

El acompañamiento y asesoramiento por parte de un tutor durante el trayecto universitario favorece la permanencia del estudiantado, en especial de quienes se encuentran en situación de discapacidad; sin embargo, en el proceso se pueden encontrar barreras como las expresadas por los tutores:

La falta de acompañamiento por un tutor o una tutora en la formación integral de los estudiantes de los programas de posgrado es uno de los principales problemas que enfrentan dependencias como los institutos de investigación de la UMSNH (PR).

El programa de tutorías es advertido como una pérdida de tiempo, en general no existe un interés real en el acompañamiento formal de los alumnos que ingresan a los posgrados (HG).

Desde la perspectiva de Álvarez (2017), la tarea fundamental de la tutoría en la universidad debe centrarse en la ayuda y orientación del alumnado en cinco apartados clave: los procesos de adaptación; los procesos de aprendizaje; el asesoramiento acerca de las fuentes de información; los procesos de toma de decisiones; y las relaciones con el entorno. De ahí que otro reto sea no solo retomar la delimitación del perfil y las funciones del docente-tutor, sino sensibilizarlos sobre la trascendencia de su labor y la responsabilidad que implica su ejercicio.

\section{IDENTIFICACIÓN DE ELEMENTOS FACILITADORES A PARTIR DE LAS HISTORIAS DE VIDA}

La intervención con estudiantes con discapacidad se realizó a través de historias de vida, mediante las cuales logramos identificar los facilitadores, que son "todos aquellos factores en el entorno de una persona que, cuando están presentes o ausentes, mejoran el funcionamiento y reducen la discapacidad" (OMS, 2001). A partir de esta definición, construimos tres categorías: facilitadores personales, facilitadores familiares y facilitadores escolares.

\section{Facilitadores familiares}

Para los estudiantes con discapacidad, el apoyo de su familia nuclear es el principal facilitador de su inclusión:

Como no puedo escribir, mi mamá me ayudaba hacer todos mis apuntes y, curso toda la secundaria a mi lado en la escuela (GA-discapacidad motriz).

Mi hermana se encontraba en la ciudad de Morelia, haciendo su carrera universitaria. Ella aceptó que me fuera con ella para que yo continuara estudiando (LF-discapacidad visual).

Asimismo, la familia extendida es otro facilitador:

Mi abuelita siempre estaba conmigo, recuerdo que en receso ella iba a la escuela a tomar el almuerzo conmigo y se quedaba a esperarme a que yo saliera de clases (DY-discapacidad visual).

Mi primo Roberto Carlos es mi sombra tanto para ir a la escuela como para ir al deporte, él me acompaña, me apoya y le doy las gracias por todo lo que hace conmigo (GA-discapacidad motriz). 
El papel de la familia es fundamental para favorecer los procesos de inclusión (social, educativa, laboral); esto se debe a que el desarrollo de habilidades y competencias personales y sociales de todos los individuos, y en nuestro caso en particular de las personas con discapacidad, estará determinado por las oportunidades brindadas por la familia a sus miembros (Sarto, 2001). De esta forma, cuando la familia se interesa, se compromete y apoya, hay más posibilidades de inclusión.

La familia, además de ser un apoyo, se puede convertir en un importante motor para salir adelante:

Mi hermano Jesús [...] también tiene discapacidad visual [...] Para mí cada paso que doy, es también pensar en él (LF-discapacidad visual).

Ahora que ya estoy en la universidad pongo todo mi empeño [...] quiero terminar mi carrera y encontrar un buen trabajo para apoyarlos [a padres y abuelos] y también a mis hermanas (DY-discapacidad visual).

El verse como los que están abriendo camino con su ejemplo a los que vienen atrás y el poder retribuir algo a sus familias se convierten en un potente catalizador en los procesos de inclusión.

\section{Facilitadores personales}

Estos facilitadores tienen que ver con características e intereses personales de los estudiantes con discapacidad que favorecen su inclusión; el esfuerzo y la dedicación son los más relevantes entre los participantes:

Siempre me ha gustado ganarme mis lugares con esfuerzo, empeño y total dedicación (LF-discapacidad visual).

Hoy comparto que fue una etapa difícil, pero la superé con entusiasmo y fuerza, tan es así que con alegría comparto, que ese semestre obtuve el primer lugar en aprovechamiento académico en el grupo (LF-discapacidad visual).

No soy un estudiante de diez, pero me esfuerzo mucho por sacar una buena calificación (GA-discapacidad motriz).

De manera general, se tiende a sobreproteger a las personas con alguna discapacidad y se frena su independencia y autonomía. Además, su inclusión al mercado laboral se ve limitada. Pese a lo anterior, otras de las características personales que sobresalen en los estudiantes con discapacidad de la UMSNH son el emprendimiento, la autonomía y la independencia, pues han logrado autoemplearse, conseguir y generar trabajo, así como vivir solos:

Yo trabajo en una empresa americana donde tengo socios, además con mis ahorros invertí para comprarme un equipo de sonido el cual llamo SONIDO SOBRE RUEDAS, donde tengo cuatro años con el equipo y también le doy empleo a personas con discapacidad [...] Me he dedicado a componer canciones; tengo 18 temas ya registrados en la Sociedad de Autores y Compositores de México y 10 temas por registrar (GA-discapacidad motriz).

Desde chico me interesé en el comercio, compraba y vendía distintos artículos. Logré ahorrar un poco y establecí en el pueblo un negocio (LF-discapacidad visual). 
Comencé a trabajar como enfermera en Morelia para apoyar a mis padres (DY-discapacidad visual).

Actualmente, estoy trabajando en Cinépolis en el área VIP. Mi labor ahí es proporcionar alimentos antes de abrir el cine, entregar órdenes, recibir y despedir a los clientes, vender promocionales tales como cubetas vasos y o juguetes y entregar menús a los clientes (GA-discapacidad motriz).

Uno de los pasos más grandes para mi inclusión fue cuando determiné que ya no podía seguir viviendo con mi hermana, que tenía que vivir solo. Aunque ella siempre es y será uno de los más grandes ángeles que Dios puso en mi camino, puesto que siempre me apoya, hoy soy más independiente (LF-discapacidad visual).

El interés que más destaca y que se convierte en un gran facilitador para la inclusión de los estudiantes con discapacidad es el deporte adaptado, en el cual se utilizan medios distintos a los habituales para permitir a los deportistas con discapacidades físicas, intelectuales o sensoriales practicar sin riesgo su deporte (Martínez-Ferrer, citado en Segura, Martínez-Ferrer, Guerra y Barnet, 2013):

Practicaba [...] un deporte llamado goalball, que consiste en cuatro jugadores por equipo con un balón con cascabeles en su interior, donde cada jugador tiene delimitada su área de defensa por relieve y uno como jugador se tiene que lanzar por la pelota ya que si cruza nuestras áreas pues es gol para el equipo contrario (LF-discapacidad visual).

En la escuela hicieron un grupo de deportistas con parálisis cerebral y yo escogí el deporte especial llamado boccia, empezamos a entrenar y en ese año hicimos un selectivo para la Paralimpiada de Puebla y quedé seleccionado; entonces fuimos a Puebla yo y mi mamá por parte de la asociación y mi papá y mi hermana nos alcanzaron allá. Fue muy bonito y muy difícil, ya que era mi primera vez en competencias nacionales, pero gracias a mi esfuerzo y el apoyo de mis padres y maestros obtuve la medalla de plata en mi categoría de BC1. De ahí en adelante me comprometí a seguir adelante; he participado en todas las Paralimpiadas y multinacionales que ha habido; he viajado mucho y conocido muchos amigos y entrenadores que son muy buenos amigos; mi vida empezó a girar alrededor del deporte (GA-discapacidad motriz).

El deporte adaptado mejora la calidad de vida de las personas en situación de discapacidad, porque incrementa las relaciones sociales, forma hábitos positivos y genera experiencias de éxito; además, contribuye a la visibilización de las personas con discapacidad y a la concientización y cambio de actitud de la sociedad sobre el tema.

\section{Facilitadores escolares}

Los profesores se convierten en facilitadores cuando estos tienen disposición para apoyar a los estudiantes con discapacidad, cuando son accesibles y flexibles en sus metodologías y evaluaciones y, sobre todo, cuando creen en ellos:

He recibido mucho apoyo por parte de mis maestros, los cuales con mi parálisis me daban la oportunidad para hacer los trabajos en casa en una computadora y se los enviaba por correo y me los calificaban y los exámenes me los hacía orales o me dejaban trabajos para la calificación (GA-discapacidad motriz).

El profesor encargado de mi grupo se dio cuenta que yo podía acceder a los contenidos con marcadores negros, con un tamaño de letra muy grande (DY-discapacidad visual). 
Gracias al apoyo de una maestra que siempre ha creído en mí [...], ella en ningún momento me dejó solo, juntos buscamos los medios y la forma de pasar la materia (LFdiscapacidad visual).

Los compañeros de clase también son mencionados como facilitadores por los estudiantes con discapacidad:

Ellos me apoyaban mucho, ya que cuando tenía que ir al baño, ellos me llevaban y les agradezco mucho (GA-discapacidad motriz).

Mis compañeros me ven como un compañero más, con una discapacidad sí, pero no observan primero la discapacidad y luego mi persona, sino de manera contraria (LFdiscapacidad visual).

Fueron un gran apoyo emocional y académico, ya que me prestaban sus apuntes [...] y me explicaban de manera individual lo que dijeron los profesores [...] fue un apoyo de pares solidarios (KE-discapacidad auditiva).

Los estudiantes con discapacidad valoran de manera positiva cuando los profesores los ponen como ejemplo para sus demás compañeros o cuando sus compañeros los ven como un ejemplo a seguir. Por ello, "convertirse en ejemplo" es otro de los facilitadores para la inclusión:

La maestra me ponía de ejemplo que yo era un alumno que cumplía en todo (LF-discapacidad visual).

Mis compañeros me buscaban porque decían que era inteligente, que tenía una gran capacidad mental y comprensiva (LF-discapacidad visual).

Para algunos de mis compañeros soy ejemplo, ya que dicen que cuando ellos ya no tienen ganas de seguir adelante se acuerdan de mí (GA-discapacidad motriz).

Los estudiantes con discapacidad valoran positivamente que las instituciones escolares tomen en cuenta sus características para brindarles la atención y los apoyos que requieren:

Cuando realicé los trámites de admisión, me llamaron para preguntarme las condiciones de mi limitación física con la finalidad de ofrecerme asistencia y un lugar más adecuado para poder realizar mi examen de ingreso. Fue un gesto muy amable y hasta cierto punto confuso, en un país en donde la sensibilidad es solo una palabra en el diccionario, te sorprende que alguien piense en tu limitación (JE-discapacidad motriz).

La atención a todos los estudiantes con discapacidad el día de examen fue excelente, porque de una u otra forma podemos sentirnos apoyados y el camino es más fácil (DYdiscapacidad visual).

\section{REFLEXIONES FINALES}

Si bien las IES tienen la responsabilidad social de facilitar la incorporación de personas en situación de discapacidad al ámbito profesional (Aquino, García e Izquierdo, 2012), no resulta fácil para las universidades transitar de una cultura homogeneizante a una inclusiva, que reconozca la diversidad como una oportunidad de enriquecimiento y no como un problema. No obstante, se cuenta con múltiples experiencias exitosas al 
respecto, en diversos países, así como en México, que permiten apreciar que, si se van generando las condiciones que posibiliten el cambio, puede lograrse paulatinamente.

Asimismo, la literatura que fundamenta las políticas, la gestión y las prácticas es amplia, lo que facilita el trabajo al respecto, e incluso, como en el caso del documento de Booth y Ainscow (2000), difundido por la Unesco, plantea dimensiones e indicadores muy claros para evidenciar qué tanto las instituciones son inclusivas, o no. Así pues, las tres dimensiones que propone dicho documento: crear culturas inclusivas, elaborar políticas inclusivas y desarrollar prácticas inclusivas, marcan claras líneas de trabajo para ir alcanzando el cambio cultural.

No obstante, se debe tener cuidado, pues no se trata solo de un simple ejercicio de reconocimiento, ya que, como lo señala Skliar (2017),

el otro es anterior a todo reconocimiento. El otro ya es, ya está, ya estuvo antes de mí. Si así no fuera, si el otro solo existiera porque lo reconozco, sería como una estatua cubierta por una tela negra en una plaza abandonada, esperando a ser descubierta. 0 como un territorio que creemos inexistente y al que damos un nombre que no es suyo. No, no es "descubrir al otro"... No, no es "nombrar al otro". Es ser llamado por él (p. 165).

De igual manera, dicho autor previene a quien quiera propiciar una cultura inclusiva no dejarse llevar por la hipocresía y la arrogancia de querer dar voz a los que se cree que no la tienen, sino de escucharlos; de otro modo, se les estaría cosificando y minimizando; no incluyendo (Rosano, 2007).

Finalmente, grandes son los retos de la UMSNH en relación con el ingreso, permanencia y egreso satisfactorio de los estudiantes con discapacidad; sin embargo, ya se han dado los primeros pasos desde distintos frentes. Resulta indispensable visibilizar y escuchar a los estudiantes en situación de discapacidad, pues ninguna decisión que influya sobre ellos debe hacerse sin su participación plena, como queda plasmado en el lema: "Nada sobre nosotros sin nosotros", que adoptó el movimiento mundial a favor de los derechos de las personas con discapacidad (Conapred, 2016). Además, se debe prestar mayor atención a las funciones de orientación en el profesorado universitario a través de la tutoría para que el estudiante con discapacidad adquiera las competencias procedimentales, conceptuales y actitudinales necesarias para su formación universitaria integral (Zamorano, 2003). De este modo, la presencia de estudiantado con discapacidad en las universidades no será algo extraordinario, sino normalizado (Álvarez, 2017).

\section{REFERENCIAS BIBLIOGRÁFICAS}

Alcantud, F. (1995). Estudiantes con discapacidad integrados en los estudios universitarios: notas para su orientación. En F. Rivas (ed.). Manual de asesoramiento y orientación vocacional (pp. 455-470) Madrid: Síntesis. Recuperado de http://sid.usal.es/9117/8-11

Álvarez, P. R. (coord.) (2017). Tutoría universitaria inclusiva. Guía de buenas prácticas para la orientación de estudiantes con necesidades educativas específicas. Madrid: Narcea.

Aquino, S. P., García, V. e Izquierdo, J. (2012). La inclusión educativa de ciegos y baja visión en el nivel superior. Un estudio de caso. Sinéctica, revista electrónica de educación, núm. 39, pp. 1-21. Recuperado de http://www.sinectica. iteso.mx/index.php?cur=39\&art=39_12 
ANUIES (2002). Manual para la integración de personas con discapacidad en las instituciones de educación superior. Recuperado de https://www.conapred.org. $\mathrm{mx} /$ documentos_cedoc/Manual_integracion_educacion_superior_UNUIES.pdf

Booth, T. y Ainscow, M. (2000). Indice de inclusión. Desarrollando el aprendizaje y la participación en las escuelas (A. López, trad.). Bristol, UK: Centre for Studies on Inclusive Education, Unesco. Recuperado de https://www.raco.cat/ index.php/Aloma/article/download/100812/154531

Camacho Real, C. y Navarro Varela, G. A. (2011). Inclusión de personas con discapacidad en la educación virtual: el caso del Bachillerato a Distancia, U de G. Revista Mexicana de Bachillerato a Distancia, vol. 5, pp. 121-137. Recuperado de http://revistas.unam.mx/index.php/rmbd/article/view/47416/42679

Castellana, M. y Salas, I. (2005). La inclusión de los estudiantes con discapacidad en la universidad: un reto para la universidad española en el nuevo espacio europeo de la educación superior. Aula Abierta, núm. 85, pp. 57-84. Recuperado de https://www.raco.cat/index.php/Aloma/article/view/100812/154531

Conapred (2016). Nada sobre nosotros sin nosotros. La Convención de Naciones Unidas sobre Discapacidad y la Gestión Civil de Derechos. Ciudad de México. Recuperado de https://www.conapred.org.mx/documentos_cedoc/Nada\%20 sobre\%20nosotros\%20sin\%20nosotros-Ax.pdf

Cruz, R. y Casillas, M. A. (2017). Las instituciones de educación superior y los estudiantes con discapacidad en México. Revista de la Educacion Superior, vol. 46, núm. 181, pp. 37-53. https://doi.org/10.1016/j.resu.2016.11.002

Del Olmo, M. (2005). Prejuicios y estereotipos: un replanteamiento de su uso y utilidad como mecanismos sociales. Revista de Educación, pp. 13-23. Recuperado de http://rabida.uhu.es/dspace/bitstream/handle/10272/1957/ b15162084.pdf?sequence $=1$

Esteban-Guitart, M., Rivas Damián, M. J. y Pérez Daniel, M. R. (2012). Empatía y tolerancia a la diversidad en un contexto educativo intercultural. Universitas Psychologica, pp. 415-426. https://doi.org/10.11144/Javeriana.upsy11-2.etdc

Fundación Universia (2016). Guía de Atención a la Discapacidad en la Universidad. Recuperado de http://www.fundacionuniversia.net/wp

Gairín, J. y Suárez, C. (2016). Inclusión y grupos en situación de vulnerabilidad. Orientaciones para repensar el rol de las universidades. Sinéctica, revista electrónica de educación, núm. 46, pp. 1-15. Recuperado de https://sinectica. iteso.mx/index.php/SINECTICA/article/view/625/751

González, A. y Farnós, J. (2009). Usabilidad y accesibilidad para un e-learning inclusivo. Revista Educación Inclusiva, vol. 2, núm. 1, pp. 49-60. Recuperado de http://www.revistaeducacioninclusiva.es/index.php/REI/article/view/26

Guajardo-Ramos, E. (2017). Educación inclusiva en la enseñanza superior. México: Editorial Académica Española.

INEGI (2019). Desciende la proporción de personas menores de 15 años de $27.5 \%$ en 2014 a 25.3\% en 2018: ENADID 2018 (comunicado de prensa]. Recuperado de https://www.inegi.org.mx/contenidos/saladeprensa/boletines/2019/EstSociodemo/ENADID2018.pdf

INEGI (2016). Datos de discapacidad en México al 2014. Recuperado de https:// www.inegi.org.mx/saladeprensa/boletines/2015/especiales/especiales2015\%20_07_1.pdf

Juárez, M. C. (2015). Integración e inclusión educativa y laboral de personas con discapacidad visual. En N. del Río. Políticas inclusivas en la educación superior 
de la Ciudad de México (pp. 90-111). Ciudad de México: Comisión de Derechos Humanos del Distrito Federal. Recuperado de https://agendasdh.cdhdf.org. $\mathrm{mx} / \mathrm{wp}$-content/uploads/2016/09/01-Pol\%C3\%ADticas-Inclusivas-en-laEducaci\%C3\%B3n-Superior-de-la-Ciudad-De-M\%C3\%A9xico.pdf

Méndez, J. M. (2015). Inclusión de alumnos con discapacidad en la Universidad Autónoma de San Luis Potosí. San Luis Potosí: RIESLP y BECENE.

Mercado-Vargas, H., Mercado-Flores, E. A. y Mercado-Flores, C. E. (2016). Cien años de la Universidad Michoacana de San Nicolás de Hidalgo, educando y formando profesionistas y ciudadanos. Revista Contribuciones a las Ciencias Sociales. Recuperado de http://www.eumed.net/rev/cccss/2016/04/michoacan.html

Moliner, 0. (2008). Condiciones, procesos y circunstancias que permiten avanzar hacia la inclusión educativa: retomando las aportaciones de la experiencia canadiense. Revista Iberoamericana sobre Calidad, Eficacia y Cambio en Educación, pp. 27-49. Recuperado de https://revistas.uam.es/index. $\mathrm{php} / \mathrm{reice} /$ article/view/5439

Moriña, A. (2008). ¿Cómo hacer que un centro educativo sea inclusivo? Análisis del diseño, desarrollo y resultados de un programa formativo. Revista de Investigación Educativa, pp. 521-538. Recuperado de https://revistas.um.es/ $\mathrm{rie} /$ article/view/94051/90661

Moriña, A. (2004). Teoría y práctica de la educación inclusiva. Málaga: Aljibe.

Novo, I. y Muñoz, J. M. (2012). Los estudiantes universitarios ante la inclusión de sus compañeros con discapacidad: indicadores basados en la teoría de la acción razonada para los estudios de economía y empresa en la Universidad de A Coruña (España). Revista Española de Orientación y Psicopedagogía, pp. 105-122. https://doi.org/10.5944/reop.vol.23.num.2.2012.11452

ONCE (2019). Tecnología y recursos adaptados. Recuperado de https://www. once.es/servicios-sociales/tecnologiayrecursosadaptados

OMS (2001). Clasificación Internacional del Funcionamiento, de la Discapacidad y de la Salud. Recuperado de https://aspace.org/assets/uploads/publicaciones/e74e4-cif_2001.pdf

Programa Erasmus (2016). Informe de análisis de necesidades (pp. 1-83). Recuperado de http://www.museproject.eu/sites/default/files/muse_informe_de_analisis_de_necesidades_1.pdf

Romero, M. R., Kitaoka, E. S. y Rodríguez, C. L. (2007). La inclusión educativa, más allá de la pobreza y las etnias, están las personas con discapacidad. Presentado en el $2^{\circ}$ Congreso Virtual Internacional sobre Desigualdad Educativa y Social (pp. 1-17). Recuperado de https://www.pag.org.mx/index.php/ PAG/article/download/141/189

Rosano, S. (2014). El camino de la inclusión educativa en Punta Hacienda (tesis de maestría). Recuperado de http://dspace.unia.es/bitstream/handle/10334/34/0050_Rosano.pdf;jsessionid=DE6DD2269DDEA388836387D E45551B26?sequence=1

Sarto, M.P. (2001). Familia y discapacidad. Presentado en el III Congreso La Atención a la Diversidad en el Sistema Educativo (pp. 1-11). Universidad de Salamanca, Instituto Universitario de Integración en la Comunidad (INICO). Recuperado de https://campus.usal.es/ inico/actividades/actasuruguay2001/5.pdf

Segura, J., Martínez-Ferrer, J., Guerra, M. y Barnet, S. (2013). Creencias sobre la inclusión social y el deporte adaptado de deportistas, técnicos y gestores de federaciones deportivas de deportes para personas con discapacidad. Revista 
Iberoamericana de Psicología del Ejercicio y el Deporte, vol. 8, núm. 1, pp. 127152. http://dx.doi.org/10.17060/ijodaep.2017.n1.v4.1037

Skliar, C. (2017). Pedagogías de las diferencias. Buenos Aires: Novoeduc.

UMSNH (2010). Plan de Desarrollo Institucional 2010-2020 (pp. 1-86). Recuperado de https://issuu.com/victorhugohuertaanguiano/docs/pdi_20102020_12.07.10

Unesco (2005). Guidelines for inclusion: Ensuring access to education for all. París. Recuperado de https://unesdoc.unesco.org/ark:/48223/pf0000140224

Vargas-Garduño, M. L., Castro, I. L. y Méndez, A. M. (2017). La innovación educativa como estrategia para promover la inclusión en las universidades. En Reflexiones sobre innovación educativa en la Universidad Michoacana de San Nicolás de Hidalgo (pp. 1-116). Morelia: UMSNH.

Zamorano, S. (2003). La tutoría en la formación de formadores. En F. Michavila y J. García (eds.). La tutoría y los nuevos modos de aprendizaje en la universidad (pp. 153-168). Madrid: UPM-Unesco. 